\title{
Fatores Associados à Ocorrência de Vibrações Articulares
}

\section{Factors Associated to the Occurrence of Joint Sounds}

Alício Rosalino Garcia1, Ronald Jefferson Martins², Cléa Adas Saliba Garbin³ ${ }^{3}$ Paulo Renato Junqueira Zuim4, Maria Lúcia Marçal Mazza Sundefeld ${ }^{5}$

\begin{abstract}
This study aimed to verify the association of gender, economic class, quality of sleep and stress with the occurrence of joint vibration. The population of this study was made up initially by 160 individuals of both sexes in the city of Piacatu, São Paulo, Brazil, which applied the Fonseca's Questionnaire, to check the degree of temporomandibular joint dysfunction (TMD). Later, individuals with moderate and severe dysfunction were classified economically through the Criterion of Economic Classification Brazil (CCEB), aswered to the translation of the Sleep Assessment Questionnaire of Toronto (SAQ) and the Social Readjustment Rating Scale (SRRS), to verify the degree of stress. There was also an examining of electrovibratographic. From the total, $37(23.1 \%)$ subjects had moderate or severe dysfunction, of which 20 (54.1\%) were female, $21(56.8 \%)$ belonged to "D Class", 29 (78.4\%) had sleep disorders, $24(64.8 \%)$ higher degrees of stress and $19(51.4 \%)$ joint vibration. There is a statistically significant association between quality of sleep and economic class in the occurrence of joint vibration.
\end{abstract}

Keywords: Temporomandibular joint. Social class. Temporomandibular joint dysfunction syndrome. Sleep disorders. Stress.

\section{Resumo}

Objetivou-se neste trabalho verificar a associação do sexo, classe econômica, qualidade do sono e estresse com a ocorrência de vibrações articulares. A população deste estudo constituiu-se inicialmente por 160 indivíduos de ambos os sexos do município de Piacatu, São Paulo, Brasil, nos quais aplicou-se o Questionário de Fonseca, para verificar o grau de DTM. Posteriormente, os indivíduos com disfunção moderada e severa foram classificados economicamente por meio do Critério de Classificação Econômica Brasil (CCEB), responderam à tradução do Questionário de Avaliação do Sono de Toronto (SAQ) e a Escala de Reajustamento Social (SRRS), para verificar o grau de estresse. Realizou-se também exame eletrovibratográfico. Do total, 37 (23,1\%) indivíduos apresentavam disfunção moderada ou severa, sendo que destes, $20(54,1 \%)$ eram do sexo feminino, $21(56,8 \%)$ pertenciam a "Classe D", 29 (78,4\%) apresentavam distúrbios do sono, 24 $(64,8 \%)$ graus mais elevados de estresse e 19 (51,4\%) vibração articular. Há associação estatisticamente significativa da qualidade do sono e classe econômica na ocorrência de vibrações articulares.

Palavras-chave: Articulação temporomandibular. Classe social. Síndrome da disfunção da articulação temporomandibular. Transtornos do sono. Estresse.

\author{
1,3,5 Professores Adjuntos da Faculdade de Odontologia de Araçatuba, \\ Universidade Estadual Paulista \\ ${ }^{2,4}$ Professores Assistentes Doutores da Faculdade de Odontologia de Araçatuba, \\ Universidade Estadual Paulista
}

Correspodência: Ronald Jefferson Martins

Endereço: R. São Paulo, no 711 - Bairro Vila Mendonça - CEP 16015-130 Araçatuba - SP, Brasil

Fone: (18) 3625-9140

E-mail: rojema@terra.com.br / rojema@foa.unesp.br

Data de Submissão: $24 / 10 / 2008$

Data de Aceite: 08/01/2010

\section{Introdução}

A prevalência dos sinais e sintomas da disfunção temporomandibular (DTM) na população em geral varia de acordo com os diferentes estudos analisados. A Academia Americana de Dor Orofacial estima que de 40 a $75 \%$ da população apresenta pelo menos um sinal da doença e $33 \%$ deles relatam pelo menos um sintoma (OKESON, 2000). A dor e as vibrações ou ruídos articulares, estão entre os sintomas e sinais mais comumente encontrados nas DTMs (BOEVER; STEENKS, 1996).

Os fatores etiológicos que levam a desenvolver DTM podem ser agrupados em três grandes grupos e parecem estar envolvidos a fatores anatômicos, incluindo a oclusão e ATM; fatores neuromusculares e fatores psicológicos (BOEVER; STEENKS, 1996). As causas de origem psicossomáticas, na qual os sintomas físicos podem ter origem psíquica, emocional ou mental devem ser consideradas (TOMMASI, 1989). Fatores emocionais, como a ansiedade e o estresse, podem desencadear hábitos parafuncionais e tensão muscular, levando ao aparecimento dos sinais e sintomas das DTMs; dentre eles a vibração articular.

Neste estudo procurou-se verificar a associação do sexo, classe econômica, qualidade do sono e estresse com a ocorrência de vibrações articulares analisadas por meio da eletrovibratografia.

\section{Revisão da Literatura}

O ruído articular é um dos primeiros sinais de desequilíbrio biomecânico da ATM. A posição em que ocorrem no ciclo de abertura e fechamento (FARRAR, 1978), a duração (GAY et al., 1987) e a intensidade (CHRISTENSEN; ORLOFF, 1992) podem indicar o estágio da patologia articular.

A identificação das vibrações articulares pode ser realizada pela palpação bidigital (PÖLLMANN, 1980), estetoscópio (MUHL et al., 1987), microfones (WIDMALM et al., 2003) e emprego de transdutores piezoelétricos (OLIVIERI et al., 1999), sendo que a prevalência de sons articulares varia conforme 0 método empregado (PÖLLMANN, 1980).

O significado clínico dos sons articulares tem sido amplamente explorado. Os ruídos crepitantes às vezes estão associados com doenças articulares degenerativas (HANSSON; NILNER, 1975). Gay e Bertolami (1988) têm sugerido que a deficiência na qualidade do líquido sinovial aumenta $o$ atrito e produz crepitação. 
Estudos epidemiológicos mostram que os sons articulares estão presentes em 34 a $65 \%$ da população em geral (GROSS; GALE, 1983; GAY; BERTOLAMI, 1988). A ausência de sons articulares não exclui a possibilidade da DTM e tem sido observado nos casos de deslocamento do disco sem redução e em articulações com extensas áreas de remodelamento articular (ERIKSSON et al., 1985), sugerindo que muitos pacientes não têm sons articulares na presença de deslocamento do disco, o que implica uma alta prevalência falso-negativa do exame clínico (MOTOYOSHI et al., 1994; PURI et al., 2006).

\section{Materiais e Métodos}

Este estudo teve o parecer favorável para sua realização do Comitê de Ética em Pesquisa em Seres Humanos, da Faculdade de Odontologia de Araçatuba-UNESP, processo FOA 2005-01719.

\section{Classificação inicial dos indivíduos conforme o grau de DTM}

O universo do estudo constituiu-se por indivíduos de ambos os sexos do município de Piacatu, São Paulo, Brasil, nos quais aplicou-se o Questionário de Fonseca et al. (1994), para verificar o grau de disfunção temporomandibular, que consiste em uma ficha clínica específica com 10 perguntas englobando anamnese e exame físico. A cada pergunta são possíveis as respostas "sim", "às vezes" e "não", às quais são atribuídos os valores "10", " 5 " e "0", respectivamente. Para a análise do questionário foram somadas as respostas "sim", "às vezes" e "não". O indivíduo foi classificado conforme o valor encontrado em "sem disfunção", "com disfunção leve", "com disfunção moderada" e "com disfunção severa" (faixas de "0 a 15", "20 a 40", "45 a 65" e "70 a 100 ", respectivamente). Selecionaram-se os indivíduos com disfunção moderada ou severa por serem os que necessitarão de tratamento.

Classificação dos indivíduos com disfunção moderada ou severa conforme classe econômica, presença de distúrbios do sono e grau de estresse

Aplicou-se nos indivíduos com disfunção moderada ou severa os seguintes questionários:

Critério de Classificação Econômica Brasil (CCEB), proposto pela Associação Brasileira de Empresas de Pesquisa (2005), a fim de classificar os indivíduos economicamente. Pelo CCEB a escolaridade do chefe da família vale de 0 a 5 pontos; os demais pontos são fornecidos pela quantidade de bens de consumo duráveis que a família possui (automóvel, televisão em cores, rádio, geladeira, freezer, máquina de lavar roupa, etc.), pela quantidade de cômodos da casa, com ênfase ao número de banheiros e pela quantidade de empregadas domésticas mensalistas que trabalham na residência.

A soma desses indicadores, ou seja, o número de pontos obtidos, distribui a população em classes, sendo a "Classe A1 (de 30 a 34 pontos)" a mais favorecida e a "Classe $\mathrm{E}$ (de 0 a 5 pontos)" a menos favorecida.

Tradução do Questionário de Avaliação do Sono elaborado pela Universidade de Toronto (Toronto Sleep Assessment Questionnaire - SAQ) (SELAIMEN et al., 2004), para verificar a qualidade e distúrbios do sono. Apresenta 19 questões referentes aos hábitos usuais de sono durante o último mês. As respostas permitidas pelo questionário apresentam pontuações de 0 a 4 (0- nunca ou não sei, 1- raramente, 2- às vezes, 3frequentemente e 4- sempre) que somadas permite classificar o indivíduo quanto à presença ou não de distúrbio do sono. $\mathrm{O}$ ponto de corte escolhido foi 16 , por ser o de maior sensibilidade $(0,73)$ e especificidade $(0,80)$. Portanto, indivíduos com escore total de 0 a 16 pontos foram classificados como "sem distúrbio do sono" e os com valores de 17 a 76 pontos como "com distúrbio do sono".
Escala de Reajustamento Social (Social Readjustment Rating Scale - SRRS) (HOLMES; RAHE, 1967), para verificar o grau de estresse. Contém uma série de 43 eventos da vida, que incluem eventos positivos, negativos, freqüentes e raros ocorridos no último ano; como por exemplo, férias, morte do cônjuge, dificuldades sexuais, etc. $\mathrm{Na}$ escala original, solicita-se ao pesquisado que assinale os eventos ocorridos dentro de um limite determinado de tempo (último mês). Em seguida, são atribuídos pesos predeterminados aos eventos assinalados, cujo somatório compõe o escore total. Para a análise do questionário foram somados os valores médios atribuídos a cada evento da vida. Caso o mesmo tenha ocorrido mais de uma vez nos últimos 12 meses, o valor foi multiplicado pelo número de ocorrências. O escore obtido determina a chance de o indivíduo apresentar alterações em sua saúde nos próximos 2 anos, dependendo da faixa em que 0 indivíduo se enquadrar (30\% - menor ou igual a 150 pontos, $50 \%$ entre 150 e 300 pontos, $80 \%$ - acima de 300 pontos). Portanto, quanto maior o número de eventos da vida relatados (escore), maior o grau de estresse.

\section{Realização de exame eletrovibratográfico}

Realizou-se também nos indivíduos com disfunção moderada ou severa exame eletrovibratográfico, para verificar a presença de vibrações articulares. Para este exame, após a limpeza com álcool da pele localizada sobre as articulações temporomandibulares direita e esquerda, posicionaram-se transdutores (acelerômetros piezoelétricos) sobre as ATMs. O sistema estava conectado a um amplificador e este ao computador. Solicitou-se, a seguir, que o pesquisado abrisse amplamente a boca e mediu-se com régua milimetrada à distância interincisal para calibrar o aparelho. Posteriormente o pesquisado foi orientado a realizar movimentos mandibulares de abertura e fechamento acompanhando um cursor presente na tela do monitor. A todos foi permitido um minuto de treinamento para acompanhar, com sincronismo, a seta indicadora do movimento mandibular Certificada a sincronia do movimento, o registro foi aceito e gravado em disco rígido e flexível. Para a análise das vibrações foi utilizado o programa SonoPak/l, versão 1.33, da Bio-Research Associates.

Para cada pesquisado a análise foi repetida por três vezes, em quatro ciclos diferentes e as médias consideradas como a quantidade de energia vibratória medida em Hertz.

A vibração localizada no pico de cada ciclo não foi considerada na análise das intensidades vibratórias, pois corresponde ao ponto de contato oclusal na posição de máxima intercuspidação; contudo, as demais vibrações ocorridas uni ou bilateralmente foram observadas, sem a preocupação de se estabelecer um limite para "ruído".

Os dados coletados foram tabulados no programa Epi Info 2000 , v. 3.2, analisados estatisticamente por meio dos Testes QuiQuadrado e Exato de Fisher com nível de significância de 5\% e apresentados em freqüências absolutas e percentuais.

\section{Resultados}

Participaram da pesquisa 160 indivíduos, sendo 61 $(38,1 \%)$ classificados "sem disfunção", $62(38,8 \%)$ "com disfunção leve", 24 (15,0\%) "com disfunção moderada" e 13 (8,1\%) "com disfunção severa", segundo o Questionário de Fonseca et al (1994).

Dos 37 (23,1\%) indivíduos com disfunção moderada ou severa que participaram das demais etapas da pesquisa, $3(8,1 \%)$ pertenciam a "Classe B1", $11(29,7 \%)$ a "Classe C", $21(56,8 \%)$ a "Classe D" e $2(5,4 \%)$ a "Classe E". A maioria, $20(54,1 \%)$ eram do sexo feminino.

Verificou-se por meio da tradução do Questionário de Avaliação do Sono de Toronto (SELAIMEN et al., 2004) que 29 $(78,4 \%)$ apresentavam distúrbios do sono e através da Escala de Reajustamento Social (HOLMES; RAHE, 1967) que 24 (64,8\%) 
relataram mais eventos da vida, portanto apresentavam maiores graus de estresse.

O exame eletrovibratográfico mostrou que $19(51,4 \%)$ dos indivíduos apresentavam vibrações em alguma posição dos ciclos de abertura e fechamento.

A ocorrência da vibração articular teve associação estatisticamente significativa com a qualidade do sono (Teste Exato de Fisher, $\mathrm{p}$ valor $=0,0254$ ) e classe econômica (Teste Exato de Fisher, $p$ valor $=0,0064)$. (Tabelas 1 e 2$)$

Tabela 1 - Relação entre a qualidade do sono e ocorrência de vibração articular.

\begin{tabular}{|c|c|c|c|c|c|c|}
\hline \multirow{3}{*}{ TORONTO } & \multicolumn{4}{|c|}{ VIBRAÇÃO } & \multirow{2}{*}{\multicolumn{2}{|c|}{ Total }} \\
\hline & \multicolumn{2}{|c|}{$\begin{array}{c}\text { Sem } \\
\text { Vibração }\end{array}$} & \multicolumn{2}{|c|}{$\begin{array}{c}\text { Com } \\
\text { Vibração }\end{array}$} & & \\
\hline & $\mathrm{n}$ & $\%$ & $\mathrm{~N}$ & $\%$ & $n$ & $\%$ \\
\hline Com & 17 & 94,4 & 12 & 63,2 & 29 & 78,4 \\
\hline Distúrbio & & & & & & \\
\hline $\begin{array}{c}\text { Sem } \\
\text { Distúrbio }\end{array}$ & 1 & 5,6 & 7 & 36,8 & 8 & 21,6 \\
\hline Total & 18 & 100,0 & 19 & 100,0 & 37 & 100,0 \\
\hline
\end{tabular}

*Teste Exato de Fisher: $p=0,0254$

Tabela 2 - Relação entre a classe econômica dos pesquisados e ocorrência de vibração articular.

\begin{tabular}{llllllll}
\hline \multirow{2}{*}{$\begin{array}{l}\text { CLASSE } \\
\text { ECONÔMICA }\end{array}$} & \multicolumn{4}{c}{\begin{tabular}{c} 
Sem \\
\cline { 2 - 5 }
\end{tabular}} & \multicolumn{2}{c}{ Vibração } & \multicolumn{2}{c}{$\begin{array}{c}\text { Com } \\
\text { Vibração }\end{array}$} & \multirow{2}{*}{ Total } \\
\hline & $\mathrm{n}$ & $\%$ & $\mathrm{~N}$ & $\%$ & $\mathrm{n}$ & $\%$ \\
\hline Classe B1 & 1 & 5,6 & 2 & 10,5 & 3 & 8,1 \\
Classe C & 2 & 11,1 & 9 & 47,4 & 11 & 29,7 \\
Classe D & 15 & 83,3 & 6 & 31,6 & 21 & 56,8 \\
Classe E & 0 & 0 & 2 & 10,5 & 2 & 5,4 \\
Total & 18 & 100,0 & 19 & 100,0 & 37 & 100,0 \\
\hline
\end{tabular}

*Teste Exato de Fisher: $p=0,0064$

Não houve relação estatística significativa entre vibração articular e estresse (Teste Exato de Fisher, $p$ valor $=0.2852$ ) e entre vibração articular e sexo (Qui-quadrado $=0.7029, \mathrm{p}$ valor $=$ 0.4018). (Tabelas 3 e 4)

Tabela 3 - Relação entre estresse e ocorrência de vibração articular.

\begin{tabular}{|c|c|c|c|c|c|c|}
\hline \multirow{2}{*}{$\begin{array}{c}\text { CHANCE DE } \\
\text { DESENVOLVER } \\
\text { PROBLEMA DE } \\
\text { SAÚDE }\end{array}$} & \multicolumn{2}{|c|}{$\begin{array}{c}\text { COM } \\
\text { VIBRAÇÃO }\end{array}$} & \multicolumn{2}{|c|}{$\begin{array}{c}\text { SEM } \\
\text { VIBRAÇÃO }\end{array}$} & \multicolumn{2}{|c|}{ TOTAL } \\
\hline & $\mathrm{n}$ & $\%$ & $n$ & $\%$ & $\mathrm{n}$ & $\%$ \\
\hline $\begin{array}{c}30 \% \text { (Escore } 150 \\
\text { ou Menos) }\end{array}$ & 8 & 61,5 & 5 & 38,5 & 13 & 100,0 \\
\hline $\begin{array}{c}50 \% \text { (Escore } \\
\text { entre } 150 \text { e } 300 \text { ) }\end{array}$ & 7 & 38,9 & 11 & 61,1 & 18 & 100,0 \\
\hline $\begin{array}{l}80 \% \text { (Escore } \\
\text { acima de } 300)\end{array}$ & 4 & 66,7 & 2 & 33,3 & 6 & 100,0 \\
\hline Total & 19 & 51,4 & 18 & 48,6 & 37 & 100,0 \\
\hline
\end{tabular}

*Teste Exato de Fisher: $p=0.2852$
Tabela 4 - Relação entre sexo e ocorrência de vibração articular.

\begin{tabular}{|c|c|c|c|c|c|c|}
\hline \multirow{3}{*}{ VIBRAÇÃO } & \multicolumn{4}{|c|}{ SEXO } & \multirow{2}{*}{\multicolumn{2}{|c|}{ Total }} \\
\hline & \multicolumn{2}{|c|}{ FEMININO } & \multicolumn{2}{|c|}{ MASCULINO } & & \\
\hline & $\mathrm{n}$ & $\%$ & $\mathrm{n}$ & $\%$ & $\mathrm{n}$ & $\%$ \\
\hline Com & 9 & 45,0 & 10 & 58,8 & 19 & 78,4 \\
\hline Vibração & & & & & & \\
\hline Sem & 11 & 55,0 & 7 & 41,2 & 18 & 21,6 \\
\hline Vibração & & & & & & \\
\hline Total & 20 & 100,0 & 17 & 100,0 & 37 & 100,0 \\
\hline
\end{tabular}

\section{Discussão}

Vários fatores estão associados à ocorrência da DTM (MARTINS et al., 2007a,b,c) e a vibração ou ruído articular é um dos principais sinais desta alteração funcional (BOEVER; STEENKS, 1996; MARTINS et al., 2007c). As mulheres apesar de serem menos sensíveis à tensão emocional que os homens, possuem maiores dificuldades para aliviar essa tensão, reflexo da cultura de nossa sociedade (WEINBERG, 1977). Além disso, tendem a ter cabeças condilianas retroposicionadas quando comparadas aos homens, 0 que pode predispô-las a deslocamentos anteriores de disco (PULLINGER et al., 1985). Também a frouxidão ligamentar poderia levar a maior ocorrência de ruído articular (OKESON, 2000). Entretanto, neste trabalho não foi verificada associação estatística significativa entre sexo e vibração articular, corroborando o resultado de outro estudo (RIEDER et al., 1983).

A situação sócio-econômica está associada com morbidade e mortalidade no mundo todo, tanto nas doenças infecciosas, como nas crônico-degenerativas (SYME; BERKMAN, 1976), sendo explicada não apenas pela falta de condições materiais usualmente associadas com a pobreza, mas também por meio de atitudes e comportamentos de risco à saúde, como exercício físico, sono, dieta e tabagismo (PAINE, 2001). Verificouse neste trabalho associação entre classe econômica e ruído articular. As classes econômicas mais baixas parecem ser favoráveis para desenvolver mais estresse, talvez por serem as mais penalizadas pelo sistema econômico do país, desenvolvendo maiores alterações na biomecânica articular.

A relação entre estresse, ansiedade, tensão e disfunções músculo esqueléticas tem sido observada (AUERBACH et al., 2001); porém, o mecanismo que une esses fatores ainda não foi descrito (ALENCAR, 1997; MARCHIORI et al., 2007). Estudo mostra que naqueles indivíduos cuja qualidade de sono não é boa, eles tendem a desenvolver DTM (MARTINS et al., 2007a). Por sua vez, observou-se em nosso trabalho associação significativa entre qualidade de sono e ruído articular. É possível que a piora na qualidade de sono, em decorrência de alterações emocionais, afeta - nível de tolerância fisiológica e o indivíduo desenvolve hiperatividade muscular por meio do apertamento dental. O aumento da sobrecarga articular oriunda do apertamento, dependendo da tolerância estrutural do líquido sinovial, pode ocasionar adesões do disco ao côndilo ou osso temporal causando danos às estruturas articulares (OKESON, 2000). As alterações articulares podem ocorrer apenas no tecido fibroso de revestimento das superfícies articulares; como acontece nas erosões, produzindo ruídos articulares com características crepitantes (ROHLIN et al., 1985). Caso a adesão seja apenas temporária, a tentativa em ampliar o movimento mandibular limitado pelo paciente, pode ocasionar alongamento dos ligamentos que une 0 disco ao côndilo ou osso temporal originando certa instabilidade do disco sobre o côndilo. Neste caso, o ruído articular é detectado apenas por meio da queixa do paciente durante o levantamento da história da doença. Após o deslocamento parcial do disco, ele pode ser detectado por meio do exame clínico.

Outra condição que leva ao aparecimento do ruído articular é o espasmo do feixe superior do pterigóideo lateral. Em 
pacientes submetidos a grandes quantidades de estresse e cujo equilíbrio mandibular não apresenta estabilidade no plano horizontal, pode ocorrer falso deslocamento do disco sobre o côndilo e o desenvolvimento de ruídos articulares de baixa intensidade no início da abertura e final do fechamento da boca (LIU et al., 1989). Esses ruídos são os de mais fácil resolução durante as terapias das DTMs (GARCIA, 2000). Neste estudo, não foi verificado associação significativa entre estresse e vibração articular, sendo possivelmente explicado pelo pequeno número de indivíduos na amostra com DTM moderada ou severa.

Segundo Spruijt e Wabeke (1995) a associação entre fatores psicológicos e sons na ATM parece depender do tipo de estudo. Quando baseado na auto-avaliação por parte dos pacientes, é sobre-estimada a importância destas variáveis na produção de ruídos articulares; em contrapartida, num estudo mais refinado, a contribuição é pequena.

Apesar da característica do ruído ser indicativo de várias doenças articulares, ele pode estar presente em pacientes com ATM assintomática e estarem ausentes em alguns casos de inflamação articular. Estudo de Garcia (2000) mostra que durante a anamnese cerca de $40 \%$ dos pacientes relatam estalo como um dos primeiros sintomas observados, o que sugere que a inflamação articular é secundária ao deslocamento do disco no estágio I. Na maioria das vezes, com o tratamento da fase aguda da patologia inflamatória ocorre um aumento das vibrações devido à diminuição dos líquidos intra-articulares e redução do espasmo do pterigóideo lateral. Quando ocorre o relaxamento deste músculo, o côndilo retorna para uma posição mais retruída dentro da fossa mandibular, o que permitirá a manutenção do disco deslocado. Durante a abertura bucal, dependendo do estágio do deslocamento, o disco retorna sobre o côndilo após a ocorrência de estalo.

Os resultados desse estudo mostram correlação com os encontrados na literatura de alta prevalência de DTM nas populações (OKESON, 2000; ABOU-ATME et al., 2006; FETEIH, 2006) e são sugestivos de que a DTM advém de alterações sistêmicas que afetam a qualidade de sono, como estresse emocional e tem como sinal mais comum o ruído articular (MARTINS et al., 2007a,b,c).

\section{Conclusão}

Baseada na metodologia empregada verificou-se que há associação estatisticamente significativa da qualidade do sono e classe econômica na ocorrência de vibrações articulares.

\section{Referências}

ABOU-ATME, Y. S.; ZAWAWI, K. H.; MELIS, M. Prevalence, intensity, and correlation of different TMJ symptoms in lebanese and italian subpopulations. J. Contemp. Dent. Pract., Cincinnati, v. 7, no. 4, p. 71-78, Sept. 2006

ALENCAR JUNIOR, F. G. P. Fatores psicológicos nas disfunções craniomandibulares: estudo da relação entre graus de disfunção e escalas de ansiedade traço-estado. 1997. 179f. Tese (Doutorado em Reabilitação Oral) - Faculdade de Odontologia, Universidade de São Paulo, Bauru.

ASSOCIAÇÃO BRASILEIRA DE EMPRESAS DE PESQUISA. Critério de classificação econômica Brasil. Disponível em: <http://www.abep.org/codigosguias/ABEP_CCEB.pdf>. Acesso em: 10 mar. 2005

AUERBACH, S. M. et al. Depression pain, exposure to stressful life events, and long-term outcomes in temporomandibular disorder patients. J. Oral Maxillofac. Surg., New York, v. 59, no. 6, p. 628633, June. 2001.
BOEVER, J. A.; STEENKS, M. H. Epidemiologia, sintomatologia e etiologia da disfunção craniomandibular. In: STEENKS, M. H.; WIJER, A. Disfunções da articulação temporomandibular do ponto de vista da fisioterapia e da odontologia: diagnóstico e tratamento. São Paulo: Ed. Santos, 1996. p. 35-43.

CHRISTENSEN, L. V.; ORLOFF, J. Reproducibility of temporomandibular joint vibrations (electrovibratography). J. Oral Rehabil., Oxford, v. 19, no. 3, p. 253-263, May 1992.

ERIKSSON, L.; WESTESSON, P. L.; ROHLIN, M. Temporomandibular joint sounds in patients with disc displacement Int. J. Oral Surg., Copenhagen, v.14, no. 5, p. 428-436, Oct. 1985.

FARRAR, W. B. Characteristics of the condylar path in internal derangements of the TMJ. J. Prosthet. Dent., St. Louis, v. 39, no. 3, p. 319-323, Mar. 1978.

FETEIH, R. M. Signs and symptoms of temporomandibular disorders and oral parafunctions in urban saudi arabian adolescents: a research report. Head Face Med., London, v. 2 , article 25, Aug. 2006.

FONSECA, D. M.; VALLE, G. B. A. L.; FREITAS, S. F. T. Diagnóstico pela anamnese da disfunção craniomandibular. RGO, Porto Alegre, v. 42, n. 1, p. 23-8, jan./fev. 1994.

GARCIA, A. R. Energia vibratória em pacientes sob tratamento de DTM. R. Assoc. Paul. Cir. Dent., São Paulo, v. 54, n. 4, p. $297-$ 301, jul./ago. 2000

GAY, T.; BERTOLAMI, C. N. The Acoustical characteristics of the normal temporomandibular joint. J. Dent. Res., Washington, v. 67 , no. 1 , p. $56-60$, Jan. 1988

GAY, T. et al. The acoustical characteristics of the normal and abnormal temporomandibular joint. J. Oral Maxillofac. Surg., Philadelphia, v. 45, no. 5, p. 397-407, May 1987.

GROSS, A.; GALE, E. N. A Prevalence study of the clinical signs associated with mandibular dysfunction. J. Am. Dent. Assoc., Chicago, v. 107, no. 6, p. 932-936, Dec. 1983.

HANSSON, T.; NILNER, M. A Study of the occurrence of symptoms of diseases of the TMJ, masticatory musculature and related structures. J. Oral Rehabil., Oxford, v. 2, no. 4, p. 313-324, Oct. 1975.

HOLMES, T. H.; RAHE, R. H. The Social readjustment rating scale. J. Psychosom. Res., Oxford, v. 11, no. 2, p. 213-218, Aug. 1967.

LIU, Z. J.; WANG, H. Y.; PU, W. Y. A Comparative electromyographic study of the lateral pterygoid muscle and arthrography in patients with temporomandibular joint disturbance syndrome sounds. J. Prosthet. Dent., St. Louis, v. 62, no. 2, p. 229-233, Aug. 1989.

MARCHIORI, A.V. et al. Relação entre a disfunção temporomandibular e a ansiedade em estudantes do ensino fundamental. Pesq. Bras. Odontoped. Clin. Integr., João Pessoa, v. 7, n. 1, p. 37-42, jan./abr. 2007.

MARTINS, R. J. et al. Clase social y trastornos del sueño. Relación con los desórdenes témporo mandibulares. Rev. Asoc. Odontol. Argent., Buenos Aires, v. 95, n. 3, p. 221-228, jun./jul. 2007a.

MARTINS, R. J. et al. Associação entre classe econômica e estresse na ocorrência da disfunção temporomandibular. R. Bras. Epidemiol., São Paulo, v. 10, n. 2, p. 215-222, jun. 2007b. 
MARTINS, R. J. et al. Relação entre classe sócio-econômica e fatores demográficos na ocorrência da disfunção temporomandibular. Ci. Coúde Disponível em: <http://www.abrasco.org.br/cienciaesaudecoletiva/artigos/artigo_int. php?id_artigo=970>._Acesso em: 20 ago. 2007c.

MOTOYOSHI M. et al. A Study of temporomandibular joint sounds: Part 1. Relationship with articular disc displacements. J. Nihon Univ. Sch. Dent., Tokyo, v. 36, no. 1, p. 48-51, Mar. 1994.

MUHL, Z. F.; SADOWSKY, C.; SAKOLS, E. I. Timing of temporomandibular joint sounds in orthodontic patients. J. Dent. Res., Washington, v. 66, no. 8, p. 1389-1392, Aug. 1987.

OKESON, J. P. Etiologia e identificação dos distúrbios funcionais no sistema mastigatório. In: Tratamento das desordens temporomandibulares e oclusão. 4 ed. São Paulo: Artes Médicas, 2000. p. 117-272.

OLIVIERI, K. A. N. et al. Join vibrations analysis in asymptomatic volunteers and symptomatic patients. Cranio, Baltimore, v. 17, no. 3, p. 176-183, July 1999.

PAINE PA. Atitudes sobre o papel de gênero e auto-avaliação de saúde em mulheres brasileiras de três grupos socioeconômicos. Est. Pesqui. Psicol., Rio de Janeiro, v. 1, n. 1, artigo 8, 2001.

PÖLLMANN, L. Sounds produced by the mandibular joint in young men: a mass examination. J. Maxillofac. Surg., Stuttgart, v. 8, no. 2, p. 155-157, May 1980.

PULLINGER A. G. et al. A tomographic study of mandibular condyle position in an asymptomatic population. J. Prosthet. Dent., St. Louis, v. 53, no. 5, p. 706-713, May 1985.

PURI, P. et al. Comparison of doppler sonography to magnetic resonance imaging and clinical examination for disc displacement. Angle Orthod., Appleton, v. 76, no. 5, p. 824-829, Sept. 2006.

RIEDER, C. E.; MARTINOFF, J. T.; WILCOX, S. A. The Prevalence of mandiblar dysfunction. Part I: sex and age distribution of related signs and symptoms. J. Prosthet. Dent., St. Louis, v. 50, no. 1, p. 81-88, July 1983.

ROHLIN, M.; WESTESSON, P. L.; ERIKSSON, L. The Correlation of temporomandibular joint sounds with joint morphology in fifty-five autopsy specimens. J. Oral Maxillofac. Surg., Philadelphia, v. 43, no. 3, p. 194-200, Mar. 1985.

SELAIMEN, C.; BRILHANTE, D.; GROSSI, M. L. Evaluation of the sleep assessment questionnaire (SAQ) in patients with temporomandibular disorders. R. Odonto Ciência, Porto Alegre, v. 19, n. 45, p. 224-32, jul./set. 2004.

SPRUIJT, R. J.; WABEKE, K. B. Psychological factors related to the prevalence of temporomandibular joint sounds. J. Oral Rehabil., Oxford, v. 22, no. 11, p. 803-808, Nov. 1995.

SYME, S. L.; BERKMAN, L. F. Social class, susceptibility and sickness. Am. J. Epidemiol., Baltimore, v. 104, no. 1, p. 1-8, July 1976.

TOMMASI, A. F. Distúrbios psicogênicos. In: Diagnóstico em patologia bucal. São Paulo: Pancast Ed., 1989. p. 645-653.

WEINBERG, L. A. An evaluation of stress in temporomandibular joint dysfunction-pain syndrome. J. Prosthet. Dent., St. Louis, v. 38, no. 2, p. 192-207, Aug. 1977.
WIDMALM, S. E.; DJURDJANOVIC, D.; MCKAY, D. C. The Dynamic range of TMJ sounds. J. Oral Rehabil., Oxford, v. 30, no. 5, p. 495-500, May 2003. 